\title{
Metacognição e Funções Executivas: Relações entre os Conceitos e Implicações para a Aprendizagem
}

\author{
Helena Vellinho Corso ${ }^{1}$ \\ Tânia Mara Sperb \\ Graciela Inchausti de Jou \\ Jerusa Fumagalli Salles \\ Universidade Federal do Rio Grande do Sul
}

\begin{abstract}
RESUMO - A pesquisa sobre aprendizagem indica que as capacidades de planejamento, monitoramento e controle são centrais ao processo de aprender e que falhas ou atraso no seu desenvolvimento estão presentes nas dificuldades de aprendizagem. Tais capacidades são definidas como metacognição em uma abordagem psicológica e como funções executivas em uma abordagem neuropsicológica. Assim, deparamo-nos com dois conceitos distintos que remetem a capacidades mentais semelhantes. Neste trabalho objetivou-se, através da revisão da literatura clássica e recente, examinar os conceitos de metacognição e de funções executivas, relacionando-os entre si e com o aprender. Como resultado, evidenciou-se que os conceitos aproximam-se em alguns aspectos e divergem em outros, e que há dados empíricos iniciais confirmando a relação entre as habilidades descritas pelos dois conceitos.
\end{abstract}

Palavras-chave: metacognição, funções executivas, cognição, aprendizagem

\section{Metacognition and Executive Functions: Relationships between Concepts and Implications for Learning}

\begin{abstract}
Research on learning indicates that the capacities of planning, monitoring and controlling one's own activity has a central role in learning, and that failure or delay in its development are present in learning disabilities. Such capacities are defined as metacognition in the psychological approach, and as executive functions in the neuropsychological approach. Thus, these two different concepts seem to refer to similar capacities. By reviewing both classic and recent literature, we aimed to examine these two concepts, relating them to each other and to learning. As a result, we found that these concepts converge in some aspects, but differ in others; and that there are some initial empirical data confirming the relationship between the skills described by both concepts.
\end{abstract}

Keywords: metacognition, executive functions, cognition, learning

O estudo sobre a aprendizagem, realizado no marco de diferentes perspectivas teóricas e no âmbito de disciplinas distintas, vem relacionando o processo de aprender às capacidades de planejamento e regulação da própria atividade em função de determinados objetivos. A Psicologia Cognitiva atribui a tal capacidade - definida como metacognição - um papel central no processo de aprendizagem (Jou \& Sperb, 2006; Son, 2007; Veenman, Van Jout-Wolters \& Afflerbach, 2006). No campo da Educação, confere-se às estratégias metacognitivas, entendidas aí como um conjunto de práticas e procedimentos, o potencial para aprimorar o processo do aprendizado (Jou \& Sperb, 2008; Martín, 2004; Monereo, Pozo, \& Castelló, 2004; Pozo, Monereo, \& Castelló, 2004).

Ao mesmo tempo, as dificuldades de aprendizagem aparecem, em diferentes estudos, relacionadas a falhas ou ao atraso no desenvolvimento dessas capacidades de planejamento, monitoramento e controle da própria atividade (Cutting, Materek, Cole, Levine, \& Mahone, 2009; Cypel, 2006; Fletcher, Lyons,

1 Endereço para correspondência: Universidade Federal do Rio Grande do Sul, Instituto de Psicologia, Rua Ramiro Barcelos, 2600, sala 114. Porto Alegre, RS. CEP 90035-003.E-mail: hvcorso@gmail.com.
Fuchs, \& Barnes, 2009; Van der Sluis, Jong, \& Van der Leij, 2006). Da perspectiva da Neuropsicologia, são as funções executivas que respondem por tais capacidades.

Ao revisar as publicações sobre aprendizagem e dificuldades de aprendizagem, deparamo-nos com dois conceitos distintos que parecem remeter a capacidades mentais semelhantes. No marco da Psicologia Cognitiva, especificamente a partir da abordagem do Processamento da Informação, é o conceito de metacognição que faz referência a essas funções que vão além da própria cognição, posto que não se relacionam a habilidades mentais específicas, mas oferecem uma organização abrangente para essas últimas (Brown, 1987; Dunlosky \& Metcalfe, 2009; Jou \& Sperb, 2006). Em uma abordagem neuropsicológica, é o conceito de funções executivas que designa essa capacidade de gerenciamento dos recursos cognitivos, capacidade essa que se relaciona de modo especial (embora não exclusivo) com o córtex pré-frontal (Cypel, 2006; Goldberg, 2002; Lezak, Howieson, \& Loring, 2004; Powell \& Voeller, 2004; Santos, 2004; Ylikoski \& Hänninen, 2003).

O presente artigo caracteriza-se como um estudo teórico que tem como primeiro objetivo examinar e relacionar o conceito psicológico de metacognição e o conceito neurop- 
sicológico de funções executivas. Para tal exame, buscamos traçar o histórico de cada um dos conceitos no contexto das disciplinas que os utilizam, bem como esquadrinhar seus significados, apontando para sua abrangência, formas de avaliação, e indicando aspectos problemáticos de um e de outro. Como segundo objetivo, buscamos enfatizar a relação de cada conceito com o processo de aprendizagem. Tratando-se de um estudo teórico, a metodologia utilizada foi a revisão de literatura. Procedeu-se ao exame de textos clássicos ou precursores nos dois temas, bem como à busca de artigos recentes (2000 a 2011; bases de dados: MEDLINE, PsycINFO, SCIENCEDIRECT; palavras-chave: metacognição, funções executivas), selecionando-se, entre esses: artigos de revisão, artigos discutindo a avaliação dos dois construtos, artigos empíricos testando a relação entre os construtos, ou a relação de cada um com aspectos da aprendizagem ou com dificuldades de aprendizagem.

De uma perspectiva educacional, ganha todo sentido e relevância a consideração das habilidades metacognitivas e das funções executivas, ao mesmo tempo em que fica clara a importante aplicação da pesquisa envolvendo tais conceitos. Relacionar os níveis de análise psicológico e neuropsicológico da aprendizagem humana e esclarecer nomenclaturas, conceitos e processos subjacentes, parecem ser passos importantes, tanto no sentido de fundamentar estratégias educacionais que incrementem o processo de aprendizagem, como no intuito de aprimorar os recursos diagnósticos e as opções terapêuticas frente às dificuldades de aprendizagem.

\section{Metacognição}

Flavell (1987) define metacognição como a cognição sobre objetos cognitivos, mas explica que o conceito poderia abranger qualquer aspecto psicológico (emoções, motivações), e inclui na definição qualquer tipo de monitoramento. Deve-se a esse autor a introdução, no âmbito da Psicologia Cognitiva, do termo metacognição (Brown, 1987; Son, 2007), que, com ele e Wellman (Flavell \& Wellman, 1977), passou a caracterizar uma área específica de pesquisa (Jou \& Sperb, 2006).

Entretanto, mesmo antes do termo ser inaugurado, a pesquisa sobre o conhecimento e o controle que as pessoas têm acerca de sua própria cognição já podia ser identificada a partir, inclusive, de perspectivas teóricas diferenciadas. Como demonstra Brown (1987), a metacognição como temática de estudo apresenta raízes históricas em diferentes teorias cognitivas ou em diferentes linhas de pesquisa. Especificamente, a autora identifica quatro problemas em Psicologia, pertencentes ao domínio da metacognição, que vinham sendo historicamente pesquisados de forma separada, por abordagens também distintas. Os relatos verbais tomados como dados seriam um primeiro problema de natureza metacognitiva, já que implicam o acesso consciente aos fatos cognitivos. A autorregulação e a abstração reflexionante são realidades psicológicas metacognitivas estudadas dentro da escola piagetiana. A noção de uma regulação exercida pelo outro surge como uma noção vigotskiana. Finalmente, a caracterização dos fatos metacognitivos como controle executivo é usada dentro de um modelo de processamento da informação.
$\mathrm{Na}$ perspectiva da abordagem do processamento da informação, os fatos metacognitivos são descritos como operações desempenhadas por um processador central ou sistema executivo capaz de realizar uma avaliação inteligente das operações de todo o sistema. Diante de um problema a ser solucionado, o processador ou executivo seria capaz de identificar e caracterizar o problema em questão, planejar estratégias adequadas, monitorar e supervisionar a efetividade dos procedimentos requisitados, e ainda avaliar dinamicamente todas essas operações (Brown, 1987).

Nesse marco teórico, a metacognição corresponde, então, a um subsistema de controle, dentro do sistema cognitivo, que tem a finalidade de monitorar, planejar e regular seus processos. Pode ser descrita como uma fase de processamento de alto nível, que permite ao indivíduo monitorar, autorregular e elaborar estratégias para potencializar sua cognição (Jou \& Sperb, 2006).

Jou e Sperb (2006) fazem referência a dois modelos de metacognição. O de Flavell (1987) apresenta quatro estruturas que se relacionam: "objetivos cognitivos" (metas relacionadas à tarefa) e "ações cognitivas" (realizações para atingir as metas) que interagem entre si e com a "experiência metacognitiva" (experiência subjetiva relacionada ao evento cognitivo) e o "conhecimento metacognitivo" (conhecimento do todo cognitivo, dividido em variáveis da pessoa, da tarefa e da estratégia). O modelo funcional de Nelson e Narens (1996), coerente com a abordagem do processamento da informação, enfatiza duas relações de fluxo de informação - a de monitoramento e a de controle - entre a instância cognitiva, ou nível objeto, e a instância metacognitiva, ou nível meta (Dunlosky \& Metcalfe, 2009; Jou \& Sperb, 2006).

Em 1987, Brown indicava que o termo metacognição poderia ser problemático, pois diferentes formas de conhecimento estariam nele sendo subsumidas. Uma das principais fontes de confusão é o fato de o termo ser aplicado a duas áreas de pesquisa distintas - o conhecimento sobre cognição (que é estável, pode ser declarado e apresenta um desenvolvimento tardio), de um lado, e a regulação da cognição (que é instável, não necessariamente passível de ser declarada e relativamente independente da idade), de outro. No mesmo ano, Flavell (1987) também apontava para a necessidade de avanços teóricos e empíricos no campo da metacognição. Por um lado, o conceito de metacognição precisava sofrer refinamento, clarificação e diferenciação. Por outro, progressos metodológicos em termos de avaliação e medidas relativas à experiência metacognitiva e ao conhecimento metacognitivo eram também indispensáveis.

Definições mais recentes parecem buscar a diferenciação necessária. Por exemplo, Dunlosky e Metcalfe (2009) propõem que metacognição não é um conceito único, mas multifacetado por natureza, destacando o conhecimento metacognitivo (conhecimento sobre a cognição), o monitoramento metacognitivo (avaliação da situação atual da atividade cognitiva) e o controle metacognitivo (regulação de algum aspecto da atividade cognitiva). Também é importante a distinção entre a metacognição explícita e a metacognição implícita. Dentro desta última, destacam-se estudos sobre o julgamento de aprendizagem, investigando a relação pre- 
ditiva entre esse julgamento e o desempenho (Kimball \& Metcalfe, 2003; Nelson, Narens, \& Dunlosky, 2004).

Desde que Flavell cunhou o termo e realizou os primeiros esforços no sentido de definir o conceito até os dias de hoje, identifica-se uma enorme quantidade de pesquisas relacionadas à metacognição. Entretanto, o incremento da pesquisa no campo da metacognição não necessariamente levou a um efetivo avanço teórico ou à clareza terminológica. Veenman et al. (2006) chamam a atenção para a proliferação de "termos metacognitivos" que vem acontecendo nos últimos anos, muitos emanados da pesquisa: crenças metacognitivas, consciência metacognitiva, sentimento de saber (feeling of knowing), julgamento da aprendizagem (judgment of learning), teoria da mente, metamemória, habilidades metacognitivas, habilidades executivas, habilidades de alta ordem, monitoramento da compreensão, estratégias de aprendizagem, entre outros, seriam termos associados à metacognição. Alguns desses termos se referem de modo geral ao conhecimento e às habilidades metacognitivas, enquanto outros são dirigidos a habilidades específicas, a certos grupos de idade, ou a tipos determinados de tarefas. Além disso, a relação entre alguns termos específicos e o conceito geral de metacognição não é definido de forma inequívoca. Em consequência, incoerências e inconsistência parecem ainda marcar, conforme os autores, o campo da metacognição.

A evolução na compreensão da metacognição relaciona-se com a evolução no entendimento de sua adequada avaliação. Também nesse campo há dificuldades. $\mathrm{O}$ exame dos instrumentos usados para avaliar a atividade metacognitiva revela a dificuldade de pesquisar a metacognição, isto é, observá-la, medi-la e manipulá-la experimentalmente (Mayor, Suengas, \& González Marqués, 1995). Os métodos mais utilizados são entrevistas, escalas de acesso metacognitivo e registros de pensamento em voz alta (autorrelatos) enquanto se executa uma tarefa cognitiva. Os dois primeiros tipos são exemplos de métodos off-line (que buscam reconstruir o processo metacognitivo depois que ele já ocorreu), enquanto o último é um método on-line (busca-se apreender o processo metacognitivo no momento em que ele está acontecendo). Ainda podem ser citados como métodos de avaliação: questionários, observações, lembrança estimulada, registro de movimentos oculares (Veenman et al., 2006). Considerando-se os métodos de avaliação de metacognição, um dos aspectos a ser determinado de modo mais preciso é qual componente da metacognição pode ser avaliado de forma efetiva por cada método, pois este pode envolver de modo geral o conhecimento metacognitivo, ou pode avaliar habilidades específicas, como monitoramento ou controle (Veenman et al., 2006).

\section{Metacognição e Aprendizagem}

Dentro de diferentes abordagens teóricas fica claro o importante papel da capacidade metacognitiva nos processos de aprendizagem e de desenvolvimento (Brown, 1987). Martín (2004) identifica em Piaget, Vigotski e na teoria do processamento da informação, apesar dos importantes aspectos em que essas teorias divergem, uma indicação semelhante: pensar, aprender e se desenvolver supõem pôr em prática processos executivos que permitam regular a própria atividade com vistas à consecução de um objetivo.

A Psicologia Cognitiva, ao elaborar modelos de processamento de informação envolvidos na aprendizagem, destaca as capacidades metacognitivas como elementos centrais do aprender. Os modelos de funcionamento cognitivo, diante de determinadas tarefas, salientam, entre outros, os processos de controle e planejamento, de seleção, organização, monitoramento e avaliação. No modelo de compreensão de texto de Kintsch e Van Dijk (1978), por exemplo, as etapas entre o input e o output comportam procedimentos metacognitivos, como verificar a coerência do que foi lido para decidir se a leitura deve prosseguir ou se é necessário recomeçá-la (Jou \& Sperb, 2008).

A compreensão leitora, de fato, parece ilustrar com clareza o papel das habilidades metacognitivas na aprendizagem, estando estas últimas entre os fatores mais fortemente envolvidos com a capacidade de compreensão de textos (Fletcher et al., 2009). O êxito na compreensão da leitura, que se constitui em ferramenta fundamental da aprendizagem formal escolar, extrapola a habilidade de decodificação, para abranger funções mentais de natureza cognitiva e metacognitiva. Talvez resida aí a origem da dissociação entre reconhecimento de palavras e compreensão leitora, já que o êxito no reconhecimento não garante a compreensão (Salles \& Corso, 2009).

Também a pesquisa educacional vem enfatizando a relação entre a aprendizagem e as capacidades metacognitivas. A pesquisa com os aprendizes experientes (expert learners), por exemplo, revelou que a presença de estratégias metacognitivas explica em grande parte a facilidade com que eles aprendem (Coll \& Onrubia, 2004). Algumas pesquisas mostram que o peso das habilidades metacognitivas pode até superar o da capacidade intelectual (Prins, Veenman, \& Elshout, 2006).

Considerando a forte relação entre metacognição e aprendizagem, e visando otimizar a aprendizagem e o rendimento escolar dos alunos, vêm sendo desenvolvidas no âmbito educacional proposições e programas instrucionais em muito apoiados no conhecimento sobre metacognição (Jou \& Sperb, 2008; Monereo et al., 2004; Pozo et al., 2004). A ideia de um currículo voltado para o objetivo de ajudar a pensar, por exemplo, propõe como eixo da organização curricular a potenciação das habilidades cognitivas e metacognitivas (Martín, 2004).

Segundo Son (2007), a Psicologia Cognitiva, de um lado, e a Educação, de outro, vêm há tempo trabalhando na busca dos princípios efetivos da aprendizagem, com vistas a aprimorar o aproveitamento acadêmico (rendimento escolar). Embora tendo trabalhado separadamente, ambas as disciplinas chegaram ao conceito de metacognição. Essa última caracterizaria, então, a possibilidade de constituir uma ponte entre as áreas. As definições estabelecidas por uma e outra área não são idênticas (a metacognição descrita pela Educação refere-se a estratégias práticas, como apontamentos e resumos), mas, de todo modo, o fato de que essas duas áreas diferentes de pesquisa, ao investigarem a aprendizagem humana, tenham chegado ao conceito de metacognição, confirma a centralidade das capacidades metacognitivas no processo de aprendizagem. 


\section{Funções Executivas}

Segundo Jurado e Rosselli (2007), um novo conceito - o de função executiva - foi descrito em sua essência pela primeira vez como o executivo central do modelo de memória de trabalho proposto por Baddeley e Hitch (1974). Na década de 1980, seria Lezak a responsável por uma primeira definição, que atribuía às funções executivas a dimensão do comportamento humano que se refere ao "como" tal comportamento é expresso, e que comportava quatro componentes - formação de objetivos, planejamento, realização do plano direcionado ao objetivo, e desempenho eficaz. Nos anos subsequentes, verificou-se uma proliferação de definições diversas para o conceito de funções executivas, assim como para os seus possíveis subcomponentes, definições essas que, a despeito das diferenças, concordam com a complexidade e importância do funcionamento executivo para o comportamento humano (Jurado \& Rosselli, 2007).

Cypel (2006) caracteriza as funções executivas como um sistema funcional neuropsicológico, composto por um conjunto de funções responsáveis por dar início e desenvolver uma atividade com um objetivo determinado. Tal sistema gerencia os recursos cognitivo-comportamentais com as finalidades de planejamento e regulação do comportamento. Um aspecto central nas diferentes definições encontradas diz respeito a essa distinção entre funções propriamente cognitivas, de um lado, e funções de segunda ordem, encarregadas de fornecer uma organização abrangente às primeiras - as funções executivas. Os diferentes recursos cognitivos e emocionais são, então, mantidos, controlados e integrados pelas funções executivas (Cypel, 2006; Goldberg, 2002; Lezak et al., 2004; Powell \& Voeller, 2004; Santos, 2004; Ylikoski \& Hänninen, 2003).

As funções executivas organizam as capacidades perceptivas, mnésicas e práxicas dentro de um contexto, com a finalidade de: eleger um objetivo; decidir o início da proposta; planejar as etapas de execução; monitorar as etapas, comparando-as com o modelo proposto; modificar o modelo, se necessário; avaliar o resultado final em relação ao objetivo inicial (Cypel, 2006). Tais processos não estão presentes apenas durante um processamento cognitivo, mas são requeridos também em decisões pessoais, e interações sociais, envolvendo, entre outros aspectos, desejo e motivação. Assim, as funções executivas abrangem o comportamento pessoal e social (Lezack et al., 2004).

Santos (2004) chama atenção para as dificuldades conceituais envolvendo as funções executivas, já que uma grande variedade de processos e funções está incluída no conceito. Possivelmente levando em conta a referida abrangência e variedade das funções executivas, alguns autores propõem sua categorização em domínios distintos. Ylikoski e Hänninen (2003) agrupam as funções executivas em quatro domínios: desejo ou vontade (antecipação, motivação, comportamento intencional), planejamento (conceitualização, tomada de decisão, desenvolvimento de esquema para executar plano), ação propositiva (programação da atividade, manutenção, integração e alternância de sequências de comportamentos, flexibilidade mental, inibição, controle da atenção e memória), e monitoramento (controle do desempenho efetivo, regulação de aspectos qualitativos do comportamento, utilização de feedback). Powell e Voeller (2004) propõem a divisão do conceito em subdomínios, referentes ao controle cognitivo (que inclui funções como memória de trabalho, controle da atenção, planejamento, monitoramento, raciocínio abstrato e solução de problemas), ao controle do comportamento (que inclui iniciativa de movimentos ou comportamentos, controle de impulso, antecipação de consequências, entre outras funções) e, ao controle emocional (que abrange modulação da excitação emocional, modulação de humor, estratégias autotranquilizadoras).

Essa distinção entre um domínio mais estritamente cognitivo e outro envolvendo aspectos mais afetivos do comportamento também é referida na literatura internacional através das designações de funções executivas "frias" e "quentes". Castellanos, Sonuga-Barke, Milha e Tannock, (2006) explicam que as primeiras seriam eliciadas diante de problemas mais abstratos, relativamente descontextualizados, enquanto as segundas seriam requeridas diante de problemas com envolvimento emocional. Ardila (2008) denomina as funções executivas ligadas ao domínio cognitivo de "funções executivas metacognitivas", e chama de "funções executivas emocionais/motivacionais" aquelas ligadas ao controle emocional. Os testes usuais de funções executivas basicamente cobrem as do tipo metacognitivo, embora a resolução dos problemas no dia a dia requeira também habilidades emocionais. Por isso, esses testes teriam uma validade ecológica limitada.

Capovilla (2006) destaca a importância tanto do desmembramento do conceito, quanto do desenvolvimento de testes que avaliem seus componentes específicos, como atenção seletiva, flexibilidade e planejamento. Algumas tarefas para avaliação das funções executivas, descritas em Kristensen (2006), são: Torre de Londres; Teste de labirintos; Teste de Stroop; Tarefas tipo go/no-go; Teste de Wisconsin; Teste de Trilhas e Provas de alternância entre tarefas. A bateria FAB (Frontal Assessment Battery), adaptada para o uso nacional por Beato, Nitrini, Formigoni e Caramelli (2007), avalia semelhanças, fluência verbal, séries motoras, instruções conflitantes, go/no-go e autonomia. A Bateria de Avaliação Comportamental da Síndrome Disexecutiva (Behavioural Assessment of the Dysexecutive Syndrome - BADS), com dados nacionais pesquisados por Armentano, Porto, Brucki e Nitrini (2009), envolve atividades da vida diária, podendo ser considerada um instrumento mais ecológico.

Como explicam Van der Sluis et al. (2006), o fato de que as funções executivas necessitam da estrutura de uma tarefa para se tornarem manifestas implica em uma série de dificuldades para o seu estudo. Um dos problemas fundamentais na medição das funções executivas seria o da "impureza" da tarefa, já que esta sempre envolve outras habilidades cognitivas não executivas, como habilidade verbal, velocidade motora, ou habilidade visuoespacial. Como as tarefas executivas são complexas (envolvem mais do que uma função executiva) e "não puras" (no sentido de envolverem muitas habilidades cognitivas), o desempenho nelas não pode ser prontamente atribuído à ausência ou presença de uma dada capacidade executiva. E, quando uma relação é observada entre o desempenho em uma tarefa executiva e o desempenho em outra medida cognitiva, não fica claro se essa relação é devida às demandas de processos executivos ou não-executivos da tarefa usada na avaliação da função executiva. 
Se a diferenciação do conceito "funções executivas" é importante para sua clareza teórica e também para sua operacionalização, a categorização das funções executivas em subdomínios distintos parece encontrar sustentação nos achados da pesquisa em neurociências. Em relação aos correlatos neuroanatomofuncionais das funções executivas, convém lembrar que, já na divisão do sistema nervoso em unidades funcionais proposta por Luria, as regiões frontais aparecem como responsáveis pelo planejamento, regulação, controle e execução do comportamento (Cosenza, 2004; Jurado \& Rosselli, 2007; Riesgo, 2006). Entretanto, os lobos frontais constituem uma grande área cerebral responsável por múltiplas funções, o que determinou sua divisão em três regiões principais: córtex pré-central, córtex pré-motor, e córtex pré-frontal, sendo que esse último está mais relacionado às funções executivas (Cosenza, 2004; Rotta, 2006; Santos, 2004). Os estudos em neuroanatomia revelam a complexidade das conexões do córtex pré-frontal, que recebe fibras de todas as áreas de associação unimodais, da área heteromodal temporo-parietal, e tem conexões recíprocas com estruturas límbicas e com o tálamo (Cosenza, 2004). Com base nessa conectividade e em características citoarquitetônicas, o córtex pré-frontal pode ser dividido em três regiões, que integram sistemas frontal-subcorticais específicos (as áreas subcorticais conexas incluem os gânglios da base, o tálamo e o cerebelo) (Powell \& Voeller, 2004).

A área pré-frontal dorsolateral recebe conexões dos lobos parietal e temporal e desempenha papel central no controle, regulação e integração das atividades cognitivas. Ela controla a distratibilidade, mantém o foco do sistema cognitivo, tanto quanto permite mudanças flexíveis desse sistema quando necessário, e está envolvida na memória e na fluência verbal. O córtex orbitofrontal é uma zona de convergência polimodal que integra diversas fontes de informação. Ele faz parte do sistema límbico e integra informações e memórias emocionais e autonômicas, estando envolvido na modulação do comportamento social, incluindo empatia, moralidade, automonitoramento e cognição social. Finalmente, o circuito cingulado anterior está envolvido no controle executivo, na atenção dividida, na detecção de erro, no monitoramento de respostas e no desencadeamento e manutenção de comportamentos contínuos apropriados. Desempenha papel central na atenção, emoção e motivação. Em resumo, as três áreas do córtex pré-frontal e suas conexões formam unidades funcionais, cada uma servindo a diferentes processos executivos (Powell \& Voeller, 2004).

Outro aspecto relevante ao se considerar as funções executivas e seus correlatos neurais diz respeito ao desenvolvimento tardio do córtex pré-frontal. Ele é a estrutura do cérebro que surgiu mais tardiamente em termos filogenéticos, sendo que a ontogênese repete esse desenvolvimento tardio (Powell \& Voeller, 2004). Ao considerar as origens evolucionárias das funções executivas, Ardila (2008) propõe que as funções executivas metacognitivas são significativamente dependentes da cultura. Seriam, provavelmente, o resultado do desenvolvimento e evolução de instrumentos de conceitualização e da linguagem oral (e escrita), em especial. Já as funções executivas motivacionais/emocionais provavelmente seriam o resultado de uma evolução biológica compartilhada com outros primatas.

\section{Déficits nas Funções Executivas e Dificuldades de Aprendizagem}

A literatura sobre dificuldades de aprendizagem, especialmente numa perspectiva neuropsicológica e neurobiológica, destaca o papel das funções executivas no processo de aprendizagem, e, consequentemente, o déficit dessas funções nos quadros de dificuldade de aprendizagem. Cypel (2006) argumenta que a atividade escolar é o protótipo de uma solicitação ambiental no sentido da autonomia e dos recursos atencionais e de organização. Tal atividade, com suas exigências em termos de determinação de objetivos, de planejamento para executá-los, de controle sobre os passos estabelecidos, demanda um desempenho eficiente das funções executivas.

Considerando a relação entre as atividades escolares e as funções executivas, Zingerevich e LaVesser (2008) verificaram que a contribuição das funções executivas para o desempenho em sala de aula de crianças autistas de alto funcionamento é mais significativa do que a contribuição do processamento sensorial da informação. Van der Sluis et al. (2006) examinaram a participação de funções executivas específicas - flexibilidade (shifting), inibição (inhibition), e atualização e monitoramento (updating) - nas habilidades de leitura, aritmética e raciocínio não verbal, em crianças entre 9 e 12 anos, verificando que é significativo o papel do monitoramento na leitura e aritmética, enquanto que o raciocínio não verbal envolve principalmente a flexibilidade.

Especificamente as dificuldades em compreensão leitora, para voltar ao exemplo citado em seção anterior, envolvem a presença de déficits em processos cognitivos identificados como subdomínios das funções executivas (Powell \& Voeller, 2004). A relação entre compreensão na leitura e memória de trabalho já foi bastante estudada (por exemplo, Friedman \& Miyake, 2004; Seigneuric, Ehrlich, Oakhill, \& Yuill, 2000). Alguns estudos concluem que é o sistema executivo central da memória de trabalho o fator diretamente relacionado com a compreensão (Carretti, Borella, Cornoldi, \& De Beni, 2009; Carretti, Cornoldi, De Beni, \& Romanò, 2005; De Beni \& Palladino, 2000; Swanson \& Jerman, 2007). Nessa mesma direção, Cutting et al. (2009) verificaram um desempenho significativamente inferior nas funções executivas entre crianças com dificuldades específicas na compreensão. Em estudo que testou a correlação entre tarefas de leitura e diferentes funções neuropsicológicas, Salles e Corso (2011) verificaram que, considerando a tarefa de compreensão leitora, as correlações significativas mais altas ocorreram com uma tarefa de funções executivas.

\section{Relações entre Metacognição e Funções Executivas - Discussão e Síntese Final}

Embora se deva considerar o comportamento, a cognição e a base neural da atividade mental humana como níveis de análise relativamente independentes, é sempre de interesse buscar as relações entre esses aspectos (Mansur \& Radanovic, 2004). Tais relações podem enriquecer reciprocamente a compreensão sobre cada um desses níveis. Nesse sentido, parece-nos procedente o estabelecimento de aproximações 
entre o conceito de "metacognição" (próprio das teorias psicológicas cognitivas) e o conceito de "funções executivas" (utilizado em Neuropsicologia), além da relação de ambos com a aprendizagem, tanto em seu padrão típico quanto patológico.

Colocando os dois conceitos em relação, pudemos encontrar aspectos em que eles se aproximam, e aspectos divergentes. Como se viu, funções executivas e metacognição descrevem as atividades mentais de planejamento, monitoramento, e controle das próprias ações. Ambos caracterizam, portanto, conceitos amplos e "compostos". Há, com certeza, o envolvimento de um conjunto de diferentes capacidades e funções para que se possa: estabelecer um objetivo, planejar a melhor estratégia para alcançá-lo, monitorar o desenvolvimento da ação naquele sentido, regular o comportamento com vistas à consecução do plano, e avaliar o resultado final.

$\mathrm{O}$ aspecto problemático identificado nas duas áreas Psicologia e Neuropsicologia - em relação a cada um dos conceitos decorre justamente de sua abrangência excessiva, que resulta em falta de precisão. Desse modo, verificamos que as duas áreas que trabalham com cada um desses conceitos não chegaram a uma forma acabada na definição de um e de outro. Há evolução na definição de ambos, sendo que tal evolução vem acontecendo no sentido de uma maior diferenciação dos domínios ou elementos que os compõem.

A evolução que o conceito de metacognição vem sofrendo diz respeito à distinção entre os aspectos de conhecimento da própria cognição e de regulação da cognição, identificando-se, então, dois processos distintos (Brown, 1987). Dunlosky e Metcalfe (2009), em sua definição, distinguiram conhecimento metacognitivo, monitoramento metacognitivo e controle metacognitivo. Segundo o modelo de Nelson e Narens (1996), monitoramento e controle caracterizam dois processos distintos e complementares dentro do aspecto regulatório da metacognição (Pannu \& Kaszniak, 2005; Souchay \& Isgrini, 2004).

Quanto ao conceito de funções executivas, a diferenciação proposta pelos autores consultados, de certa forma, enfatiza mais explicitamente a separação entre os aspectos mais emocionais ou mais cognitivos do planejamento e do controle da atividade. Essa tendência ficou evidenciada na separação entre funções executivas consideradas "frias" e aquelas consideradas "quentes" (Castellanos et al., 2006), na classificação de Powell e Voeller (2004), que estabelece um subdomínio de controle emocional, e na classificação de Ylikoski e Hänninen (2003), que inclui a "vontade" entre os diferentes domínios que compõem as funções executivas.

Como se vê, embora ambos os conceitos apresentem esse aspecto multifacetado, diferentemente do conceito de metacognição, o de funções executivas extrapola o domínio estritamente cognitivo, abrangendo também o domínio afetivo ou emocional do comportamento e da cognição. Entretanto, metacognição em sua conceituação mais abrangente e na ideia inicial de Flavell (1987) contempla o conhecimento que o sujeito tem sobre todos os fenômenos psicológicos, como emoção e motivação.

Verifica-se, ainda, que o aspecto complexo e multidimensional de ambos os conceitos impõe dificuldades à pesquisa (operacionalização dos conceitos) e à prática clínica (diagnósticos diferenciais), o que remete à questão da avaliação. Nesse sentido, verificaram-se diferenças entre os métodos e instrumentos utilizados para investigar as funções executivas e aqueles usados na avaliação da metacognição. Enquanto se utilizam testes em uma avaliação direta, no caso das funções executivas, como o teste de trilhas e a torre de Londres, no caso da metacognição são utilizadas medidas indiretas, como escalas de autoavaliação e informes verbais. Se há diferenças nas formas de avaliação, nos dois casos verifica-se a demanda por aprimoramentos. Em relação à observação da metacognição, considera-se que esses métodos oferecem limites à sua medição e manipulação, sendo que ainda falta padronização nos inventários utilizados para avaliar cada componente metacognitivo. Além disso, é apontada a necessidade de estabelecer quais são os componentes metacognitivos efetivamente avaliados por cada método. Em relação à avaliação das funções executivas, verifica-se a busca no sentido da determinação de quais são as funções específicas envolvidas em cada teste, bem como a investigação do peso dos aspectos propriamente executivos nas diferentes tarefas utilizadas. Também é importante observar que a avaliação da metacognição se apoia em domínios específicos do conhecimento, como leitura, matemática, física, etc. Contrariamente, a avaliação neuropsicológica de funções executivas segue o modelo experimental de pesquisa. Desse modo, a pesquisa em metacognição estaria mais de acordo com a proposta de validade ecológica.

Portanto, nem no plano teórico, e, consequentemente, nem no nível empírico, encontramos formas acabadas e consensuais em termos de definições ou de formas de avaliação. Parece tratar-se de conceitos em construção, referidos que estão a realidades mentais complexas. Contemplar essa complexidade na definição do conceito, atentando para a multiplicidade de elementos em jogo, sem descuidar do aspecto integrativo que marca a capacidade metacognitiva e as capacidades executivas, apresenta-se como um desfio à Psicologia e à Neuropsicologia.

Importa relacionar metacognição e funções executivas não apenas levando em conta os aspectos da formalização ou da construção dos conceitos pelos campos em que eles foram concebidos. Como vimos, sob esse aspecto encontram-se semelhanças e diferenças. Cabe também perguntar se metacognição e funções executivas teriam uma relação possível de ser demonstrada empiricamente. Isto é, será que habilidades metacognitivas ou componentes da metacognição correspondem às funções executivas, ou a componentes das funções executivas, de modo que, possivelmente, desempenhos de participantes em tarefas de metacognição e funções executivas se correlacionariam?

Fernández-Duque, Bird e Posner (2000) propõem que metacognição e funções executivas estão intimamente relacionadas. Comparam o modelo de metacognição de Nelson e Narens (1996) ao modelo de funcionamento executivo de Norman e Shallice (1986), fazendo corresponder o nível meta e o nível objeto do primeiro modelo ao sistema executivo e aos esquemas do segundo modelo. Identificam o mesmo funcionamento de monitoramento e controle em ambos os modelos, e afirmam que, apesar da similaridade conceitual, a pesquisa em metacognição e funções executivas vêm acontecendo de forma separada. 
Souchay e Isgrini (2004) citam o trabalho de Fernandez-Duque et al. (2000), concordando com a crítica acerca da forma independente com que a pesquisa em ambos os campos vinha acontecendo, e sugerindo a necessidade de que a relação entre metacognição e funções executivas seja melhor examinada. Os autores revisam pesquisas que sugerem uma ligação direta entre o funcionamento do lobo frontal e a metamemória, sugerindo que as funções de metamemória são mediadas pelo mesmo sistema neural que também dá suporte às funções executivas. Um dos objetivos do trabalho de Souchay e Isgrini (2004) foi determinar se o funcionamento executivo e o controle metacognitivo podem ser relacionados. Idosos e adultos jovens tiveram seu desempenho avaliado em tarefa envolvendo controle de metamemória e em testes neuropsicológicos para avaliação de funções executivas, tendo sido verificada uma correlação parcial significativa entre os resultados de ambas as avaliações. Além disso, a análise de regressão hierárquica indicou que o declínio no controle da metamemória relacionado com a idade pode ser resultado das limitações das funções executivas associadas com o envelhecimento.

A revisão da literatura, tanto em metacognição quanto em funções executivas, revela que, nos anos recentes, tem havido um esforço de investigar empiricamente as possíveis relações entre os dois conceitos. Componentes metacognitivos - ou construtos associados com o domínio metacognitivo - de um lado, e funções executivas, de outro, são verificados conjuntamente em diferentes participantes de pesquisa (adultos, crianças, grupos clínicos), sugerindo que pelo menos parte da pesquisa em metacognição e em funções executivas não mais acontece de forma isolada. Na direção do trabalho de Souchay e Isgrini (2004), citado acima, Perrotin, Isgrini, Souchay, Clarys e Taconnat (2005) encontraram uma relação específica entre os escores de testes executivos e os de uma tarefa de monitoramento de metamemória (episodic feeling-of-knowing). Em 2008, alguns desses mesmos autores (Perrotin, Tournelle, \& Isgrini, 2008) verificaram que a maior parte da variação na acurácia do julgamento sobre a própria memória (feeling-of-knowing) deve-se ao funcionamento executivo. Fahie e Symons (2003) encontraram uma forte relação entre o desempenho em tarefa de teoria da mente e em testes de funções executivas em crianças com problemas de comportamento e de atenção. Os autores concluem que o funcionamento executivo e a teoria da mente podem ser indicadores de déficits metacognitivos que estão por trás dos problemas sociais apresentados pelas crianças da amostra. Benson e Sabbagh (2010), ao considerarem a pesquisa sobre a relação entre o desenvolvimento da teoria da mente e o desenvolvimento do funcionamento executivo em crianças, fazem um exame teórico aprofundado acerca da natureza dessa relação, bem como das bases neurodesenvolvimentais que poderiam explicá-la.

Além da relação entre metacognição e funções executivas, estabelecemos como objetivo deste trabalho examinar a relação entre os dois conceitos e a aprendizagem. A implicação das capacidades metacognitivas e executivas no processo de aprendizagem parece ser uma realidade inconteste. $\mathrm{O}$ estudo desenvolvido até aqui deixa clara a presença fundamental da metacognição e das funções executivas no processo de aprendizagem, assim como seu comprometimento nos quadros de dificuldade de aprendizagem. Seleção, organização, elaboração, retenção e transformação da informação relevante são requeridas a todo o momento diante do conteúdo escolar. Mesmo nos níveis escolares mais iniciais, as capacidades metacognitivas ou executivas devem estar presentes. É assim que, como vimos, a compreensão a partir da leitura de textos é garantida por recursos atencionais e de memória, bem como estratégias de organização e realização de raciocínios, entre outros aspectos. Nesse sentido, verifica-se que a capacidade de compreensão leitora é um palco perfeito para a observação das relações entre as habilidades metacognitivas e a aprendizagem. Da mesma forma, num sentido negativo, evidências (Cutting et al., 2009; Fletcher et al, 2009) revelam déficits em funções executivas entre alunos com dificuldade em compreensão leitora.

A presença de disfunções executivas nas dificuldades de aprendizagem indica a necessidade de incluir o exame das funções executivas na avaliação psicológica, neuropsicológica e psicopedagógica das dificuldades de aprendizagem. Também a intervenção terapêutica precisa abranger as habilidades metacognitivas e/ou as funções executivas. Se planejamento e regulação são elementos centrais do processo de aprendizagem, e se estão comprometidos em quadros de dificuldade de aprendizagem, então compreender essas capacidades mentais, descritas tanto pela Psicologia Cognitiva como pela Neuropsicologia, é indispensável à prática escolar e aos profissionais que se ocupam do aprender, em uma perspectiva preventiva ou terapêutica.

\section{Referências}

Ardila, A. (2008). On the evolutionary origins of executive functions. Brain and Cognition, 68, 92-99.

Armentano, C. G. C., Porto, C. C., Brucki, S. M. D., \& Nitrini, R. (2009). Study on the Behavioural Assessment of the Dysexecutive Syndrome (BADS) performance in healthy individuals, Mild cognitive impairment and Alzheimer's disease: A preliminary study. Dementia \& Neuropsychologia, 3(2), 101-107.

Baddeley, A .D., \& Hitch, G. (1974). Working memory. In G.H. Bower (Ed.), The psychology of learning and motivation: Advances in research and theory Vol. 8 (pp. 47-89). New York: Academic Press.

Beato, R. G., Nitrini, R., Formigoni, A. P., \& Caramelli, P. (2007). Brazilian version of the Frontal Assessment Battery (FAB): Preliminary data on administration to healthy elderly. Dementia \& Neuropsychologia, 1, 59-65.

Benson, J. E., \& Sabbagh, M. A. (2010). Theory of mind and executive functioning. In P. D. Zelazo, M. Chandler, \& E. Crone (Eds.), Developmental Social Cognitive Neuroscience (pp. 63-80). New York: Psychology Press.

Brown, A. L. (1987). Metacognition, executive control, selfregulation, and other more mysterious mechanisms. In F. E. Weinert \& R. Kluwe (Eds.), Metacognition, motivation, and understanding (pp. 1-16). Hillsdale, New Jersey: Lawrence Erlbaum Associates.

Capovilla, A. (2006). Desenvolvimento e validação de instrumentos neuropsicológicos para avaliar funções executivas. Avaliação Psicológica, 5(2), 239-241. 
Carreti, B., Borella, E., Cornoldi, C., \& De Beni, R. (2009). Role of working memory in explaining the performance of individuals with specific reading comprehension difficulties: A metaanalysis. Learning and Individual Differences, 19, 246-251.

Carretti, B., Cornoldi, C., De Beni, R. \& Romanò, M. (2005). Updating in working memory: a comparison of good and poor comprehenders. Journal of Experimental Child Psychology, 91, 45-66.

Castellanos, F. X., Sonuga-Barke, E. J. S., Milha, M. P., \& Tannock, R. (2006). Characterizing cognition in ADHD: beyond executive dysfunction. Trends in Cognitive Sciences, 10(3), 117-123.

Coll, C., \& Onrubia, J. (2004). Inteligência, inteligências e capacidade de aprendizagem. In C. Coll, A. Marchesi, \& J. Palácios (Eds.), Desenvolvimento psicológico e educação psicologia da educação escolar, vol. 2 (pp. 131-144). Porto Alegre: Artmed.

Cosenza, R. M. (2004). Bases estruturais do sistema nervoso. In V. M. Andrade, F. H. Santos, \& O. Bueno (Eds.), Neuropsicologia Hoje (pp. 37-59). São Paulo: Artes Médicas.

Cutting, L. E., Materek, A., Cole, C. A. S., Levine, T. M., \& Mahone, E. M. (2009). Effects of fluency, oral language, and executive function on reading comprehension performance. Annals of Dyslexia, 59, 34-54.

Cypel, S. (2006). O papel das funções executivas nos transtornos da aprendizagem. In N. Rotta, L. Ohlweiler, \& R. Riesgo (Eds.), Transtornos da aprendizagem - Abordagem neurobiológica e multidisciplinar (pp. 375-387). Porto Alegre: Artmed.

De Beni, R., \& Palladino, P. (2000). Intrusion errors in working memory tasks: are they related to reading comprehension ability? Learning \& Individual Differences, 12, 131-143.

Dunlosky, J., \& Metcalfe, J. (2009). Metacognition: a textbook for cognitive, educational, life span and applied psychology. Thousand Oaks, California: SAGE Publications.

Fahie, C. M., \& Symons, D. K. (2003). Executive functioning and theory of mind in children clinically referred for attention and behavior problems. Applied Developmental Psychology, 24, 51-73.

Fernández-Duque, D., Bird, J. A., \& Posner, M. I. (2000). Executive attention and metacognitive regulation. Consciousness and Cognition, 9, 288-307.

Flavell, J., \& Wellman, H. (1977). Metamemory. In R. V. Kail \& J.W. Hagen (Eds.), Perspective on the development of memory and cognition (pp. 3-33). Hillsdale, New Jersey: Lawrence Erlbaum Associates.

Flavell, J. H. (1987). Speculations about the nature and development of metacognition. In F. E. Weinert \& R. Kluwe (Eds.), Metacognition, motivation, and understanding (pp. 1-16). Hillsdale, New Jersey: Lawrence Erlbaum Associates.

Fletcher, J. M., Lyons, G. R., Fuchs, L. S., \& Barnes, M. A. (2009). Transtornos de aprendizagem: da identificação à intervenção. Porto Alegre: Artmed.

Friedman, N. P., \& Miyake, A. (2004). The reading span test and its predictive power for reading comprehension ability. Journal of Memory \& Language, 51, 136-158.

Goldberg, E. (2002). O cérebro executivo: lobos frontais e mente civilizada. Rio de Janeiro: Imago.

Jou, G. I., \& Sperb, T. M. (2006). A Metacognição como estratégia reguladora da aprendizagem. Psicologia: Reflexão e Crítica, 19(2), 177-185.
Jou, G. I., \& Sperb, T. M. (2008). Leitura compreensiva: o processo instrucional. Linguagem e Ensino, 11(1), 145-177.

Jurado, M. B., \& Rosselli, M. (2007). The elusive nature of executive functions: a review of our current understanding. Neuropsychology Review, 17, 213-233.

Kimball, D., \& Metcalfe, J. (2003). Delaying judgments of learning affects memory, not metamemory. Memory-and-Cognition, 31(6), 918-929.

Kintsch, W., \& Van Dijk, A. (1978). Toward a model of text comprehension and production. Psychological Review, 85(5), 363-394.

Kristensen, C. H. (2006). Funções executivas e envelhecimento. In M. A. M. P. Parente (Ed.), Cognição e envelhecimento (pp. 97-111). Porto Alegre: Artmed.

Lezak, M. D., Howieson, D. B., \& Loring, D. W. (2004). Neuropsychological assessment (4 ed.). New York: Oxford University Press.

Mansur, L. L., \& Radanovic, M. (2004). Neurolinguística. Princípios para a prática clínica. São Paulo: Edições inteligentes.

Martín, E. (2004). Ensinar a pensar por meio do currículo. In C. Coll, A. Marchesi, \& J. Palácios, (Eds.), Desenvolvimento psicológico e educação - Transtornos de desenvolvimento e necessidades educativas especiais - psicologia da educação escolar, vol 3 (pp. 309-329). Porto Alegre: Artmed.

Mayor, J., Suengas, A. \& González Marqués, J. (1995). Estrategias metacognitivas: aprender a aprender y aprender a pensar. Madrid: Editorial Síntesis.

Monereo, C., Pozo, J. I., \& Castelló, M. (2004). O ensino de estratégias de aprendizagem no contexto escolar. In C. Coll, A. Marchesi, \& J. Palácios (Eds.), Desenvolvimento psicológico e educação - psicologia da educação escolar, vol. 2 (pp. 161176). Porto Alegre: Artmed.

Nelson, T., Narens, L., \& Dunlosky, J. (2004). A revised methodology for research on metamemory: Pre-judgment recall and monitoring (PRAM). Psychological-Methods, 9(1), 53-69.

Nelson, T., \& Narens, L. (1996). Why investigate Metacognition? In J. Metcalfe \& A. P. Shimamura (Eds.), Metacognition. Knowing about knowing (pp. 1-27). Cambridge, Mass.: MIT Press.

Norman, D. A., \& Shallice, T. (1986). Attention to action: Willed and automatic control of behaviour. In R. J. Davidson, G. E. Schwartz, \& D. Shapiro (Eds.), Consciousness and SelfRegulation: Advances in Research and Theory, vol. 4 (pp. 1-18). New York: Plenum Press.

Pannu, J. K., \& Kaszniak, A.W. (2005). Metamemory experiments in neurological populations: a review. Neuropsychology Review, 15(3), 105-30.

Perrotin, A., Isgrini, M., Souchay, C., Clarys, D., \& Taconnat, L. (2005). Episodic feeling-of-knowing accuracy and cued recall in the elderly: evidence for double dissociation involving executive functioning and processing speed. Acta Psychologica, 122, 58-73.

Perrotin, A., Tournelle, L., \& Isgrini, M. (2008). Executive functioning and memory as potential mediators of the episodic feeling-of-knowing accuracy. Brain and Cognition, 67, 76-87.

Pozo, J. I., Monereo, C. \& Castelló, M. (2004). O uso estratégico do conhecimento. In C. Coll, A. Marchesi, \& J. Palácios (Eds.), Desenvolvimento psicológico e educação - psicologia da educação escolar. vol. 2 (pp. 145-160). Porto Alegre: Artmed. 
Powell, K. B., \& Voeller, K. K. S. (2004). Prefrontal executive function syndromes in children. Journal of Child Neurology, 19, 785-797.

Prins, F. J., Veenman, M. V. J., \& Elshout, J. J. (2006). The impact of intellectual ability and metacognition on learning: new support for the threshold of problematicity theory. Learning and Instruction, 16, 374-387.

Riesgo, R. S. (2006). Anatomia da aprendizagem. In N. Rotta, L. Ohlweiler, \& R. Riesgo (Eds.), Transtornos da aprendizagem - Abordagem neurobiológica e multidisciplinar (pp. 21-42). Porto Alegre: Artmed.

Rotta, N. (2006). Dispraxias. In N. Rotta, L. Ohlweiler, \& R. Riesgo (Eds.), Transtornos da aprendizagem: Abordagem neurobiológica e multidisciplinar, (pp. 207-220). Porto Alegre: Artmed.

Salles, J. F., \& Corso, H. V. (2009). Relação entre leitura de palavras isoladas e compreensão de leitura textual em crianças. Letras de Hoje, 44(3) 28-35.

Salles, J. F., \& Corso, H. V. (2011). Funções neuropsicológicas relacionadas ao desempenho em leitura em crianças. In L. M. Alves, R. Mousinho, \& S. A. Capellini (Eds.), Dislexia: novos temas, novas perspectivas (pp. 107-129). Rio de Janeiro: WAK Editora.

Santos, F. H. dos (2004). Funções executivas. In V. M. Andrade, F. H. Santos, \& O. Bueno (Eds.), Neuropsicologia Hoje (pp. 125-134). São Paulo: Artes Médicas.

Seigneuric, A., Ehrlich, M. F., Oakhill, J. \& Yuill, N. (2000). Working memory resources and children's reading comprehension. Reading and Writing, 13, 81-103.

Son, L. K. (2007). Introduction: a metacognition bridge. European Journal of Cognitive Psychology, 19(4), 481-493.
Souchay, C., \& Isgrini, M. (2004). Age related differences in metacognitive control: role of executive functioning. Brain and Cognition, 56, 89-99.

Swanson, H. L., \& Jerman, O. (2007). The influence of working memory on reading growth in subgroups of children with reading disabilities. Journal of Experimental Child Psychology, 96, 249-283.

Van der Sluis, S., De Jong, P. F., \& Van der Leij, A. (2006). Executive functioning in children, and its relations with reasoning, reading, and arithmetic. Intelligence, 35, 427-449.

Veenman, M. V. J., Van Jout Wolters, B. H. A. M., \& Afflerbach, P. (2006). Metacognition and learning: conceptual and methodological considerations. Metacognition Learning, 1, 3-14.

Ylikoski, R., \& Hänninan, T. (2003). Assessment of executive function in clinical trials. International Psychogeriatrics, 15, 219-224.

Zingerevich, C., \& LaVesser, P. D. (2008). The contribution of executive functions to participation in school activities of children with high functioning autism spectrum disorder. Research in Autism Spectrum Disorders, 3, 429-437.

Recebido em 23.06.2010

Primeira decisão editorial em 11.07.2011

Versão final em 12.07.2012

Aceito em 12.07.2012 